[0212-7199 (2005) 22: 5; pp 235-237] ANALES DE MEDICINA INTERNA Copyright $@ 2005$ ARAN EDICIONES, S.L.

An. Med. INTERNA (Madrid) Vol. 22, N. ${ }^{\circ}$ 5, pp. 235-237, 2005

\title{
Isquemia intestinal en la enfermedad de Buerger
}

\author{
J. CORDOBÈS GUAL, R. RIERA VÁZQUEZ, O. MERINO MAIRAL, E. MANUEL- \\ RIMBAU MUÑOZ, P. LOZANO VILARDELL, M. COMPANY CAMPINS ${ }^{1}$
}

Servicio de Angiología y Cirugía Vascular. 'Servicio de Anatomía Patológica. Hospital Universitario Son Dureta. Palma de Mallorca

\author{
BUERGER'S DISEASE WITH INTESTINAL ISCHEMIC INVOLVE- \\ MENT
}

\section{RESUMEN}

La enfermedad de Buerger es una arteritis que afecta de forma predominante a pacientes jóvenes con gran habito tabáquico. La sintomatología isquémica a nivel de extremidades superiores e inferiores es la forma más habitual de presentación. La afectación visceral es muy poco frecuente y a menudo se infravalora. Presentamos el caso clínico de una mujer joven de 44 años con enfermedad de Buerger y afectación intestinal, y realizamos una revisión de la literatura de pacientes con enfermedad de Buerger que presentan afectación visceral.

La afectación intestinal en la enfermedad de Buerger es extremadamente infrecuente y su diagnóstico precoz difícil, por eso ante un paciente con enfermedad de Buerger que presenta dolor abdominal de etiología no aclarada, debe evaluarse la posibilidad de una isquemia visceral. La arteriografía diagnóstica y una laparoscopia abdominal exploradora precoz ayudaran a delimitar la necrosis intestinal y el riesgo de complicaciones. En aquellos casos en que la situación hemodinámica del paciente no lo permita se procederá a la realización de una laparotomía con carácter de urgencia.

PALABRAS CLAVE: Enfermedad de Buerger. Isquemia mesentérica. Aortografía.

\begin{abstract}
Buerger's disease is uncommon arterial disease that affects mainly young people with heavy smoking history. Ischemic symptoms of upper and lower extremities are clearly defined as the most common kind of presentation. Visceral arteries are rarely affected. We report a case of a 44 years old young female with Buerger's disease and mesenteric ischemic involvement, and a revision of the literature about Buerger's disease with visceral affection. Due to the extremely rarity of the intestinal involvement of Buerger's disease, the early diagnosis is difficult, for these reason all patient affected by Buerger's disease who presents intestinal symptoms should be carefully evaluated. Aortography and early laparoscopic revision are very important to limit ischemic intestinal injury and later complications. If hemodynamic instability is present emergency laparotomy at the operating theatre is recommended.
\end{abstract}

KEY WORDS: Buerger's disease. Mesenteric ischemia. Aortography.

Cordobès Gual J, Riera Vázquez R, Merino Mairal O, Manuel-Rimbau Muñoz E, Lozano Vilardell P, Company Campins M. Isquemia intestinal en la enfermedad de Buerger. An Med Interna (Madrid) 2005; 22: 235-237.

\section{INTRODUCCIÓN}

La tromboangeítis obliterante o enfermedad de Buerger es una vasculopatía inflamatoria muy poco frecuente. Su etiología es desconocida y se observa en gente joven con importante hábito tabáquico. Afecta a las arterias de pequeño y mediano calibre con una patrón de distribución distal, sobretodo en extremidades. A pesar que la afectación de arterias viscerales es aún menos frecuente existe algún caso descrito en la literatura (1).

Presentamos el caso clínico de una paciente con enfermedad de Buerger y afectación de troncos viscerales.

\section{CASO APORTADO}

Mujer de 44 años de edad que ingresó en el servicio de Medicina Interna por presentar cuadro de dolor abdominal y diarreas asociadas de características líquidas sin productos patológicos. La paciente presentaba un hábito tabáquico de 2 paquetes/día y alergias al metamizol, keterolaco y prostaglandinas.

Como antecedentes patológicos destacaban enfermedad inflamatoria pélvica, isquemia crónica en extremidad superior izquierda en forma de dolor con claudicación del brazo a mínimos esfuerzos asociando fenómeno de Raynaud. También presentaba antecedentes de isquemia crónica en extremidad inferior izquierda en forma de dolor de reposo sin lesiones tróficas. En la arteriografía de miembros supe-

Trabajo aceptado: 27 de diciembre de 2004

Correspondencia: Jordi Cordobès Gual. C/ Archiduque Luis Salvador, 113, 2º B. 07004 Palma de Mallorca. e-mail: Corgual@yahoo.es. 
riores se objetivó una oclusión de la arteria humeral con recanalización a nivel de una arteria cúbito-interosea de características hipoplásicas, mientras que la arteriografía de miembros inferiores mostraba una oclusión de la arteria poplitea con recanalización a nivel de arterias distales de características hipoplásicas.

Se realizó un estudio de trombofilia (proteina $\mathrm{C}$ y S, antitrombina III, anticuerpos antifosfolípido, Factor V de Leyden) así como pruebas inmunológicas (velocidad de sedimentación, Factor reumatoide, anticuerpo antinuclear, anticuerpo anticentromero y anti-scl70), que resultaron negativas descartándose un estado de hipercoagulabilidad o una enfermedad reumatológica. El ecocardiograma transesofágico realizado no mostró alteraciones. Con todos estos antecedentes se hizo el diagnóstico de enfermedad de Buerger. Para la isquemia de extremidades superiores se procedió a la realización de una simpatectomía torácica por videotoracoscopia con franca mejoría clínica. Respecto a la isquemia de miembros inferiores y ante la imposibilidad de tratamiento revascularizador se optó por una actitud conservadora.

En la exploración física presentaba regular estado general, con un abdomen blando y depresible doloroso difusamente a la palpación. La exploración vascular mostró una obstrucción humeral en extremidad superior izquierda y una obstrucción femoro-poplitea en extremidad inferior derecha, no presentaba lesiones tróficas; el resto de la exploración fue anodina.

Los hallazgos de laboratorio mostraron un hematocrito del $43 \%$, una leucocitosis de $31.600 \mathrm{~mm}^{3}$ con desviación izquierda $(92 \%$ de polimorfonucleares), VSG de 66, los parámetros de función hepática y renal fueron normales al igual que los reactantes de fase aguda. La gasometría, electrocardiograma, radiografía de tórax y de abdomen fueron normales.

Con la sospecha clínica de gastroenteritis aguda se ingresó a la paciente procediéndose a la instauración de sueroterapia y reposo intestinal. Los coprocultivos y parásitos en heces resultaron negativos. Durante su estancia hospitalaria presentó empeoramiento clínico con aumento de dolor abdominal y distensión abdominal. Se realizó un TAC abdominal que resultó inespecífico. Dados los antecedentes de vasculopatía de la paciente y ante la sospecha de isquemia mesentérica se realizó una arteriografía selectiva de troncos viscerales que mostró permeabilidad del tronco celiaco y mesentérica superior con oclusión de arteria ileal, ileo-cólica y mesentérica inferior (Fig. 1).

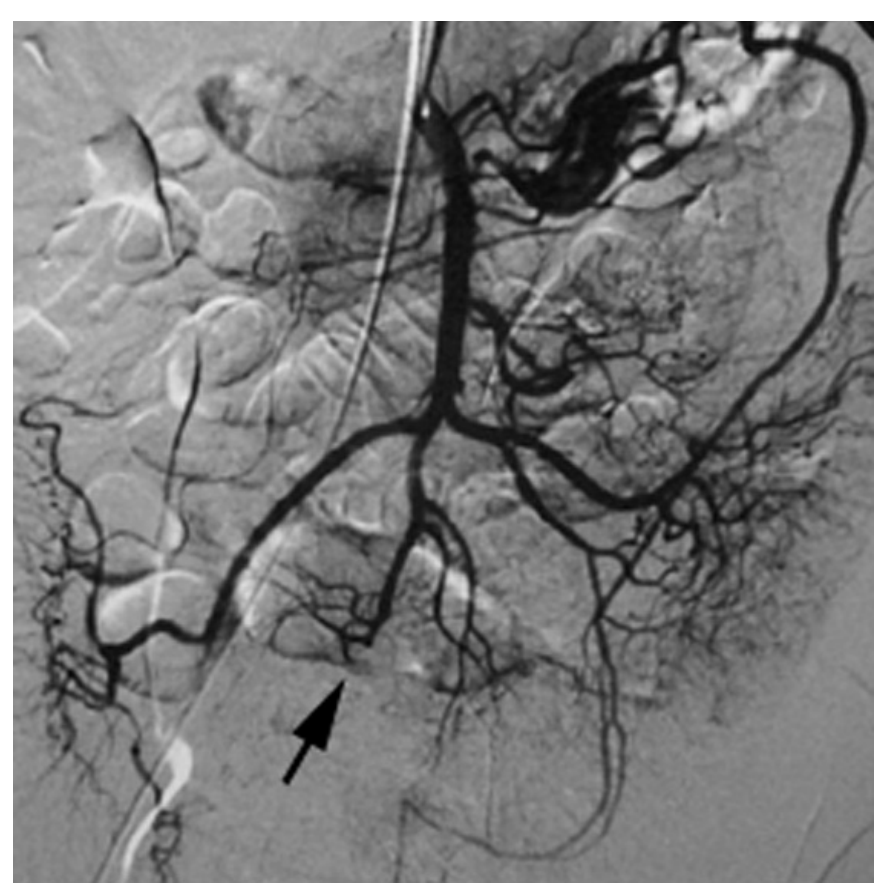

Fig. 1. Permeabilidad del tronco celiaco con oclusión de las ramas ileal e ileo-cólica de la arteria mesentérica superior.
Ante el hallazgo de una posible isquemia mesentérica por oclusión de pequeño vaso sin afectación de troncos principales, se decidió instaurar tratamiento médico mediante reposo intestinal, analgesia y nutrición parenteral.

La paciente presentó una mala evolución con deterioro progresivo de su estado general, aumento de la distensión y del dolor abdominal, aparición de nauseas, vómitos y acidosis metabólica. Dada la mala evolución clínica se procedió a la realización de una laparotomía exploradora objetivándose una oclusión intestinal con estenosis severa del intestino delgado y lesiones segmentarias difusas de aspecto isquémico, sin afectación de intestino grueso, por lo que se procedió a la realización de una resección intestinal y anastomosis termino-terminal primaria. El examen histopatológico mostró vasos con trombo en fase de recanalización e infiltración inflamatoria transmural constituyda por linfocitos, siempre con la estructura de la pared vascular conservada.

La paciente presentó un postoperatorio tórpido con dehiscencia parcial de la sutura primaria termino-terminal intestinal que precisó de una nueva laparotomía con nueva resección intestinal y anastomosis termino-terminal. La evolución posterior fue dentro de la normalidad siendo dada de alta con buena tolerancia oral y hábito deposicional normal.

\section{DISCUSIÓN}

La enfermedad de Buerger o tromboangeïtis obliterante es una vasculopatía que afecta las arterias y venas de pequeño y mediano calibre con un patrón de distribución distal, sobretodo a nivel de extremidades inferiores y superiores. A pesar de que su etiología sigue siendo desconocida, su relación con el hábito tabáquico está claramente demostrada (1). Algunos grupos sugieren una asociación con el antígeno linfocitario humano inclinándose por una predisposición genética (2). El diagnóstico es clínico apoyado por las pruebas de imagen, de laboratorio y en algunos casos, por los hallazgos histopatológicos. A diferencia del resto de vasculitis, los marcadores inmunológicos son negativos (3). Afecta predominantemente a varones jóvenes con gran hábito tabáquico, con una distribución de 8 a 1, aunque se ha visto un aumento de la incidencia en mujeres los últimos años, probablemente debido al aumento del tabaquismo en este grupo $(4,5)$.

Ocasionalmente se han descrito casos aislados de afectación de sectores arteriales atípicos en esta enfermedad, como el cerebral, coronario, renal y mesentérico $(6,7)$. La afectación de troncos viscerales es muy poco común y en la literatura médica existen escasamente descritos 30 casos de isquemia mesentérica secundaria a la enfermedad de Buerger (8-13). Su presentación clínica es del todo inespecífica, generalmente en forma de dolor abdominal sin un factor etiológico aclarado, lo que dificulta el diagnóstico y éste se realiza tras la exclusión de otras patologías. Debe realizarse el diagnóstico diferencial con enfermedades del colágeno (esclerodermia, síndrome de CREST), vasculitis como la panarteritis nodosa; también con enfermedades embólicas, estados de hipercoagulabilidad y aterosclerosis $(1,13)$. Nuestro paciente no presentaba ninguno de los factores de riesgo aterosclerótico excepto el consumo de tabaco, las pruebas de laboratorio descartaban una colagenosis, vasculitis o un estado de hipercoagulabilidad y el ecocardiograma excluía la posibilidad de un origen embólico.

El patrón de afectación arteriográfico en vasos viscerales es similar al de las extremidades. Éste preserva los principales troncos digestivos, afectando a sus ramas principales, y a dife- 
rencia de la afectación de extremidades no presenta un grado tan elevado de presencia de colaterales supletorias (14), como se observa en nuestro caso clínico. El intestino delgado se afecta en forma de lesiones segmentarias, afectándose con menos frecuencia el intestino grueso. El patrón histopatológico del segmento resecado puede ayudar en gran medida a confirmar el diagnóstico de enfermedad de Buerger, diferenciano esta entidad de la aterosclerosis y otras vasculitis sistémica (1). La presentación histopatológica típica característica de la tromboangitis obliterante se presenta en estadios en fase aguda y muestra una lesión oclusiva, con trombo inflamatorio y gran cantidad de celulas en la pared de los vasos, con preservación de la estructura normal de la pared de los vasos incluyendo la lámina elástica interna.

La mortalidad en los casos con afectación intestinal en la tromboangeïtis obliterante es elevada dado el alto riesgo de necrosis intestinal, perforación y peritonitis, siendo primordial realizar el diagnóstico precoz en los casos de sospecha de isquemia mesentérica en un paciente con enfermedad de Buerger. Se debe realizar una cuidadosa evaluación de aquellos pacientes jóvenes, fumadores y con antecedentes de afectación arterial por enfermedad de Buerger en otros sectores, que presentan un cuadro clínico de dolor abdominal no etiquetado $(3,9)$. La realización de una arteriografía y la cirugía abdominal laparoscópica precoz ante la sospecha de una isquemia mesentérica secundaria a la afectación visceral por enfermedad de Buerger, ayudarán a evitar una necrosis intestinal extensa así como a disminuir el riesgo de complicaciones. En aquellos casos con inestabilidad hemodinámica se realizará una laparotomía exploradora sin esperar al resultado de la arteriografía diagnóstica.

\section{Bibliografía}

1. Matsushita M, Shionoya S, Matsumoto T. Urinary cotinine measurement in patients with Buerger's disease: Effects of active and passive smoking on the disease process. J Vasc Surg 1992; 14: 53-58.

2. McLoughlin GA, Helsby CR, Evans CC, et al. Association of HLA-A9 and HLA-B5 with Buerger's disease. Br Med J 1976; 2: 1165-1166.

3. Kobayashi M, Kurose K, Kobata T, Hida K, Sakamoto S, et al. Ischemic intestinal involvement in a patient with Buerger disease: Case report literature and review. J Vasc Surgery 2003; 38: 170-174.

4. Mills JL, Porter JM: Buerger's disease (tromboangiitis obliterans). Ann Vasc Surg 1991; 5: 570-572.

5. Olin, JW. Current concepts: Thromboangitis Obliterans (Buerger's Disease). N Engl J Med 2000; 34: 864-869.

6. Donatelli F, Triggiani M, Nascimbene S, et al. Thromboangiitis obliterans of coronary and intestinal thoracic arteries in a young woman. J Thorac Cardiovasc Surg 1997; 113: 800-2.

7. Michail PO, Filis KA, Delladetsima JK, Koronarchis DN, Bastounis EA. Thromboangiitis obliterans (Buerger's disease) in visceral vessels confirmed by angiographic and histological findings.Eur J Vasc Endovasc Surg 1998; 16: 445-8

8. Siddiqui MZ, Reis ED, Soundararajak, Kerstein MD. Buerger's disease affecting mesenteric arteries A rare cause of intestinal ischemia. Vasc Surg 2001; 35: 235-238.

9. Cho YP, Kwon YM, Kwon TW, Kim GE. Mesenteric Buerger's disease. Ann Vasc Surg 2003; 17: 221-223.

10. Michail PO, Filis KA, Delladetsima JK, Koronarchis DN, Bastounis EA. Thromboangiitis obliterans (Buerger's disease) in visceral vessels confirmed by angiographic and histological findings. Eur J Vasc Endovas Surg 1998; 16: 445-448.

11. Schellong SM, Bernhards J, Ensslen F, Schafers HJ, Alexander K. Intestinal type of thromboangiitis obliterans (Buerger's disease). J Intern Med 1994; 235: 69-73.

12. Kempczinski RF, Clarck SM, Blebea J, Koelliker DD, Fenoglio-Preiser C. Intestinal ischemia secondary to thromboangiitis obliterans. Ann Vasc Surg 1993; 7: 354-358.

13. Cappel MS, Seibold JR. Mesenteric trombosis associated with anticardiolipin antibodies in a patient with systemic lupus erythematosus. Am J Gastroenterol 1992; 87: 520-522.

14. Borlaza G, Rapp R, Weatherbee L, Demetropoulas K. Visceral angiographic manifestation of thromboangiitis obliterans. South Med J 1979; 72: 1609-1611. 